\title{
SULLE EQUAZIONI IRREDUCIBILI DI GRADO PRIMO \\ RISOLUBILI PER RADICALI
}

Nota del prof. F. Giudioe, a Palermo.

(Sedula del 13 marzo 1987)

Le cose che espongo sono conosciute; spero turtavia che la semplicita delle dimostrazioni acquisti importanza alla presente Nota.

I. Se $p$ è primo, le radici d' un' equazione irreducibile, del grado $p$ risolubile per radicali sono tutti, e soltanto, i valori che una particolare

$$
a_{0}+R^{\frac{2}{p}}+a_{2} R^{\frac{2}{p}}+\ldots+a_{p-1} R^{\frac{p-t}{p}}=\zeta\left(R^{\frac{1}{p}}\right)
$$

la per la multiformita dei radicali che contiene. (*)

Indicando con $\omega$ una radice imaginaria $p^{\text {ma }}$ dell' unith; le radici d'una tale equazione potranno quindi esser date da:

$$
\begin{gathered}
x_{z}=a_{0}+\omega^{2} R^{\frac{1}{p}}+a_{2} \omega^{2 t} R^{\frac{2}{p}}+\ldots+a_{p-1} \omega^{(r-1) t} R^{\frac{p-1}{p}}=\xi\left(\omega^{2} R^{\frac{1}{p}}\right) \\
z=0,1,2, \ldots, p-1 .
\end{gathered}
$$

Per una qualunque

si avrà cosi :

$$
\varphi\left(x_{0}, x_{1}, x_{2}, \ldots, x_{p-1}\right)
$$

$\varphi\left(x_{0}, x_{1}, \ldots, x_{p-1}\right)=\varphi\left[\xi\left(R^{\frac{1}{p}}\right), \xi\left(\omega R^{\frac{1}{p}}\right), \ldots, \xi\left(\omega^{p-1} R^{\frac{1}{p}}\right)\right]=\Psi\left(R^{\frac{1}{p}}, \ldots\right)$.

A particolari sostituzioni delle $x$ contenute in $\varphi$ corrisponderanno particolari cambiamenti di valore dei radicali contenuti in $\Psi$ e viceversn.

(") Vedi: A bel, Giuvres, Tome second, Note XVIII - Kronecker, Mo. natshericht der Konigl. Akad. der Wissenchaften, Berlin, März 1879. 
Un cambiamento di valore dei radicali contenuti in $\zeta\left(R^{\frac{i}{p}}\right)$ avr: adunque per effetto una sostituzione delle $x$; questa sposterd 0 non sposterà $x_{0}$.

Supponiamo che $x_{0}$ sia sostituita da $x_{2} ;$ a questa sostituzione corrisponde il semplice cambiamento di $R^{\frac{1}{p}}$ in $\omega^{2} R^{\frac{1}{p}}$, e questo porta di conseguenza il cambiamento di $\omega^{m} R^{\frac{1}{p}}$ in $\omega^{m+\lambda} R^{\frac{1}{p}}$ eppero di $x_{m}$ in $x_{m+\lambda}$ dove ad ogni indice maggiore di $p$ devesi sostituire il minimo resto positivo relativo al divisore $p$. Avremo quindi, fra gli indici $z$ delle $x_{q}$, la sostituzione :

$$
|z, z+\lambda| \quad(\bmod . p)
$$

ossia, fra le $x_{i}: \quad\left(x_{0}, x, x_{2 \lambda}, \ldots, x_{\left.(\beta-1)^{\lambda}\right)}\right)$

Supponiamo ora che $x_{0}$ non sia spostata e sia invece sostituita $x_{\mu} \mathrm{da}$ $x_{v}$. A questa sostituzione corrisponde il semplice cambiamento di wh in $\omega$ '. Sia $x-i$ l'unica soluzione della congruenza $\mu x \equiv \mathrm{I}(\bmod . p)$. Il cambiamento di $\omega^{\mu}$ in $\omega^{\nu}$ porta di conseguenza quello di $\omega,=\omega^{\mu / 4}$, in $\omega^{\nu}$ ossia in $\omega^{\prime}$ se $j$ i il minimo resto positivo di vi pel divisore $p$. Verra cosi cambiata $\omega^{m}$ in $\omega^{\prime m}$; a questo cambiamento corrisponde la sostituzione di $x_{j m}$ ad $x_{m}$. Avremo quindi fra gli indici $z$ la sostituzione

$$
|z, j z| \quad(\bmod . p)
$$

che dd una sostituzione regolare delle $p-1$ radici $x_{1}, x_{2}, \ldots, x_{1-1}$, composta di $\rho$ cicli dell'ordine $\frac{p-1}{\rho}$ dove $\frac{p-I}{\rho}$ e l'esponente a cui appartiene $j$ relativamente al modulo $p$.

Segue che: Se $\varphi$ appartiene al gruppo delle sostituzioni lineari intere, fra gli indici $\pi$

$$
\begin{gathered}
G=|z, \beta z+\gamma| \\
\beta=1,2, \ldots, p-1 \quad \gamma=0,1,2, \ldots, p-1
\end{gathered}
$$


resterh essa inalterata comunque si mutino i valori dei radicali contenuti in $\xi\left(R^{\frac{2}{p}}\right)$, e sarà per ciò esprimibile razionatmente per $\mathrm{i}$ coefficienti dell'equazione.

Abbiamo cosi il teorema :

Se un'equazione irreducibile di grado primo is risolubile per radicali, le funzioni delle radici $x_{z}$ appartenenti al gruppo delle sostituzioni lineari intere fro gli indici z sono esprimibili razionalmente per mezzo dei coeffcienti dell'equazione, le radici essendo prese in ordine opportuno.

Corollario. - Le equazioni irreducibili di grado primo risolubili per radicali sono equazioni di $G$ alo is $\left(^{*}\right)$. Il loro gruppo, relativo agli indici delle $x_{1}$, è il gruppo delle sostiluzioni lineari intere od ì sollogruppo di questo come avviene per le equazioni Abeliane cle formano un caso particolare di quelle di Galois.

Se le $x$. sono indipendenti, ogni funzione appartenente al gruppo $G$ che es dell' ordine $p \times(p-1)$ ha $\frac{p !}{p(p-1)}$ valori. Per $p=2,3$ si ha $\frac{p !}{p(p-1)}=$ I; mentre per $p>5$ siha $\frac{p !}{p(p-1)}=(p-2) !>$ I.

Abbiamo quindi anche il corollario:

Le equazioni irreducibili di grado primo non minore di s, epperó anche quelle di grado composto maggiore di s, non sono risolubili per radicali.

Siccome s'è visto che ogni cambiamento di valore nei radicali che non consista nel porre $\omega^{\lambda} R^{\frac{1}{p}}$ in luogo di $R$ ha per effetto una sostituzione regolare, d'ordine $\frac{p-1}{p}$ fra le $p-1$ radici $x_{1}, x_{2}, \ldots, x_{p-1}$, si puó dedurre che:

Nell'espressione della radice ollre dei radicali esterni che sono necessariamente del grado $p$ entrano solamente radicali aventi per indiie un divisore $d i p-\mathrm{I}$.

(*) A bel (Euvres complites, t. Il, p. 270) aveva detto che un'equazione irreducibile di grado primo e risolubile per radicali se una sua radice es esprimibile razionalmente per altre due, ma non aveva enunciata la proposizione inversa. 